\title{
Progress in the Treatment of Acute Ischemic Stroke, Current Challenges and the Establishment of Clinical Decision-Making System
}

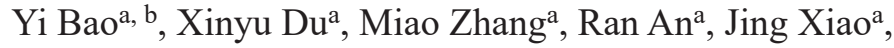 \\ Xiaodong Liu ${ }^{\mathrm{a}}$, Guangjian $\mathrm{Liu}^{\mathrm{a}}$
}

\begin{abstract}
Ischemic stroke often occurs in middle-aged and elderly people, leading to brain tissue ischemia, hypoxia and necrosis. The clinical manifestations are a series of neurological deficits, such as aphasia, hemiplegia and disturbance of consciousness, with high morbidity, mortality, disability rate, recurrence rate and multiple complications. This article aims to review current treatment advances, analyze current challenges and propose coping strategies. The literature on stroke treatment and the latest technological progress were reviewed. Combined with clinical and epidemiological to analyze the current challenges, the coping strategies were proposed before, during and after thrombolysis. Early intravenous thrombolysis and bridging treatment can restore blood perfusion in time and save the ischemic penumbra of brain tissue. However, the current proportion of patients receiving thrombolytic therapy is very low. The main challenges are as follows: easy to miss the time window, door-to-needle time is too long and there is a lack of understanding of the safety and efficacy of thrombolysis, especially the hemorrhagic transformation. A clinical decision-making system is established for stroke rescue by improving the popularization rate of stroke thrombolytic therapy, optimizing the green channel process of stroke and improving the executive ability of clinicians, to shorten the rescue time. Advanced imaging techniques are used to identify potential patients for thrombolysis. Acute intravascular bridge therapy is used to improve the efficacy of thrombolysis. Screening before thrombolysis, timely thrombolytic therapy, reexamination after thrombolysis and active response to hemorrhagic transformation can effectively improve the safety and acceptability of treatment.
\end{abstract}

Keywords: Acute ischemic stroke; Thrombolysis; Bridge therapy; Hemorrhagic transformation

Manuscript submitted June 4, 2019, accepted August 7, 2019

aDepartment of Neurology, Taihe Hospital Affiliated to Hubei University of Medicine, Shiyan City, Hubei Province, China

${ }^{b}$ Corresponding Author: Yi Bao, Department of Neurology, Taihe Hospital Affiliated to Hubei University of Medicine, Shiyan City, Hubei Province, China. Email: karlbaoyi@163.com

doi: https://doi.org/10.14740/jnr541

\section{Introduction}

Acute ischemic stroke (AIS) accounts for $70-80 \%$ of strokes, with high morbidity, mortality, disability rate, recurrence rate and multiple complications. It often occurs in middle-aged and elderly people [1-3].

Acute cerebrovascular occlusion leads to brain tissue ischemia, hypoxia and necrosis. The clinical manifestations are a series of neurological deficits such as aphasia, hemiplegia and disturbance of consciousness. The internal carotid artery occlusion may have hemiplegia, hemianopia and partial sensory disturbance, and aphasia can also occur in the dominant hemisphere. Vertebral-basal artery occlusion leads to interruption of blood supply to the occipital lobe, thalamus, corpus callosum, cerebellum and brainstem, especially in the brainstem (respiratory, circulatory center and descending nerve fiber bundles are concentrated here). If not treated in time, the mortality rate is as high as $80-90 \%$, seriously affecting the quality of life and endangering life $[4,5]$.

In addition, acute cerebral infarction patients are often left with severe neurological deficits which are life-long. As the population ages and the lifestyle changes, acute stroke has become a disease of first disability and second mortality. According to statistics, only in China, the annual incidence is up to 120 $-180 / 100,000$, and the mortality rate is up to $60-120 / 100,000$. More seriously, the age of onset is getting younger in recent years [6-8].

\section{Pathogenesis, Pathologic Process and Thera- peutic Targets of Stroke}

Brain tissue has almost no glucose or oxygen reserves, and is therefore very sensitive to ischemia and hypoxia. In order to maintain the normal nerve function of the brain tissue, a constant supply of blood must be provided. In AIS cases, the pathophysiological mechanism of neuronal necrosis is very complex, mainly involving energy failure, loss of ion balance, intracellular $\mathrm{Ca}^{2+}$ overload, excitatory neurotoxicity, activation of free radicals, release of cytokines, disruption of bloodbrain barrier, activation of glial cells, inflammatory responses, etc. This series of cascade events synergistically lead to apoptosis and necrosis. However, cerebrovascular occlusion does 
not immediately lead to cerebral infarction, and the specific development trend is closely related to the severity of ischemia and ischemic time $[9,10]$.

After cerebral arterial thrombosis, the brain tissue in the infarct center area is irreversibly damaged, while the surrounding tissue is in a low perfusion state. The brain tissue between electrical failure and membrane failure can maintain the basic function of the ion pump and the basic structure of the cells, and have the dual possibility of returning to normal tissue and progressing to infarct tissue, namely the ischemic penumbra (IP). According to the study, 1.9 million nerve cells are permanently lost in function in this area every minute, and the recovery time of cerebral perfusion is delayed by $30 \mathrm{~min}$, and the neurological recovery rate is reduced by $10 \%$. After $6 \mathrm{~h}$ of ischemic stroke, there are still $70 \%$ of the IP, and $44 \%$ of the IP after $18 \mathrm{~h}$. In IP, there are a large number of brain cells in the state of dormancy or semi-dormancy, which is a dynamic process of evolution. If blood supply is restored, the development of infarction can be prevented. Therefore, the treatment of cerebral infarction focuses on the rescue of IP, and time is the brain [11-13]. However, the onset time of some patients is unknown. The DAWN trial and the DEFUSE-3 trial found that some patients had better 90-day functional outcomes for endovascular treatment than untreated patients, even though they exceeded the thrombolysis time window[14].

At present, for the evaluation of IP, imaging is often performed by diffusion-weighted imaging (DWI), perfusionweighted imaging (PWI) and susceptibility-weighted imaging (SWI). DWI shows an irreversible cytotoxic edema lesion (i.e. infarct core); PWI shows the hypoperfusion area of cerebral ischemia, reflecting the sensitivity of brain tissue to ischemia. Studies have shown that a mismatch between a larger PWI lesion and a smaller DWI lesion is considered an IP image. SWI shows abnormal vascular changes in and around the ischemic foci, contrast enhanced between venous vessels and surrounding tissues can show collateral circulation vessels [15-18]. There have been clinical trials (EXTEND: NCT01580839; POSITIVE: NCT01852201) to use advanced neuroimaging to select patients whose symptoms appear greater than the thrombolytic window, but less than $24 \mathrm{~h}$, and conduct the experiment in order to benefit more patients $[14,19,20]$.

\section{Current Progress in Thrombolysis and Bridging Therapy}

Effective measures are taken within the treatment time window, and the IP tissue can be restored in time to save the neurons, reduce the infarct size and improve the nerve function. In the super early stage, the removal of thrombosis is the most important measure to restore blood perfusion in the infarct area, significantly improving patient survival, reducing mortality and disability and improving prognosis. Molecular studies have demonstrated the advantages of reperfusion: the activation of heat shock protein 70 and the mitogen-activated protein kinase pathway reduces intravascular glutamate expression and membrane aspartate transport receptor activity in ischemic brain tissue, thereby reducing the hyperexcitability damage to brain tissue. The restored blood flow activates the $\mathrm{Na}^{+}$channels on the cell membrane and reduces $\mathrm{Ca}^{2+}$ influx and reduces intracellular $\mathrm{Ca}^{2+}$ overloading through $\mathrm{Na}^{+}-\mathrm{Ca}^{2+}$ competitive inhibition. The above mechanisms work together to significantly shrink the IP around the cerebral infarction lesions and retain most of the normal cerebral cortex functional areas $[21,22]$.

Currently, a number of clinical trials (MR CLEAN, ESCAPE and EXTEND-IA) have shown that in addition to intravenous thrombolysis (IVT), there are intravascular treatments: arterial thrombolysis (IAT), combined arterial and venous thrombolysis (ICT + IAT), microcatheter and microcatheter thrombolysis, balloon dilatation, stent-assisted thrombectomy, thrombolysis, ultrasound-assisted thrombolysis and the combination of various techniques. By using images to select the appropriate patients and time window concept the vascular recanalization rate has been significantly improved, and a large number of patients have benefited [14, 23-25].

Comprehensive treatments in the stroke unit (SU) include: antiplatelet aggregation therapy, anti-coagulation, defibration, strengthening lipid-lowering, dilating blood vessels, improving microcirculation, removing oxygen free radicals, establishing collateral circulation and preventing complications. In terms of establishing collateral circulation, studies have shown that butylphthalide can promote the establishment of collateral circulation around the ischemic region, increase arterial blood flow velocity and increase blood supply to the ischemic area. At the same time, it can improve intracellular mitochondrial membrane stability, enhance the activity of ATP enzyme in mitochondria, stabilize the mitochondrial structure and play a role in reducing the death of neurons. In addition, neuroprotection, traditional Chinese medicine, hyperbaric oxygen and mild hypothermia have also been included in the guidelines.

IVT is currently the only clinical solution with evidencebased medicine for the treatment of cerebral infarction. Recombinant tissue plasminogen activator (rt-PA) has become the preferred drug recommended in the treatment guidelines for AIS. Thrombolytic therapy within $3-4.5 \mathrm{~h}$ of onset can effectively improve the neurological deficits, and becomes the gold standard for the treatment of AIS. The advantages are as follows: simple technical equipment, quick and convenient time for drug administration, easy to master operation technology, relatively small trauma, low cost, and patients are easy to accept. The disadvantages are as follows: the occlusion rate of the internal carotid artery and middle cerebral artery (MCA) is low; a large dosage of medicine and low local blood drug concentration; the possibility of concomitant hemorrhage transformation and systemic adverse reactions is high. What's worse, the treatment window is only $3-4.5 \mathrm{~h}$, and most patients do not benefit $[26,27]$.

Arterial thrombolysis refers to the most direct method to quickly restore cerebral blood flow by using Seldinger technology, digital subtraction angiography (DSA) and local administration to the infarct site to promote thrombolysis. The advantages are as follows: it can determine the location, degree and vascular compensation of vascular lesions, with high selectivity; it can maintain a relatively high drug concentration in the embolus, and have a high recanalization rate; it can reduce systemic thrombolytic concentration, reduce the risk of bleed- 
ing, with better safety; treatment time window can be extended to $6 \mathrm{~h}$, while patients with infarction of basilar artery, posterior circulation of brain and anterior circulation with better collateral circulation can extend the time window of thrombolysis appropriately, benefiting more patients. The disadvantages are as follows: it can only reach the proximal end of the occlusion vessel; the amount of local thrombolytic drugs is too large (the drug concentration in the infarct site is nine times that of IVT) and can increase the risk of intracranial hemorrhage; what's worse, it has high equipment requirements, complicated operation, high cost, and is difficult to promote [28, 29].

Arteriovenous combined thrombolysis refers to the practice of total cerebral angiography on the basis of IVT, followed by arterial thrombolysis if residual thrombolysis is found, taking into account the advantages of simple and rapid IVT and high recanalization rate of arterial thrombolysis, and its effectiveness and safety are relatively high, so it has been highly valued by scholars $[30,31]$.

Thrombolysis combined with ultrasound refers to the use of ultrasonic pulse to fracture emboli during thrombolysis. It increases the contact area of emboli and drug, promotes the dissolution of thrombus and makes good blood vessel dredging, which has become a hot spot of current research [32, 33].

Intravascular thrombus extraction is achieved by using the thrombolytic device combined with balloon stents, which enables the thrombolytic drugs to have greater contact area with emboli, rapidly increases the local drug concentration in the embolic artery, reduces the dosage of thrombolytic drugs and shortens the time of thrombolysis. It has the characteristics of high vascular recirculation rate and high safety [34].

Solitaire AB stent thrombectomy can directly remove thrombotic tissue from the body. Roth et al reported that patients with cerebral infarction were treated with Solitaire AB stent. The mean vascular opening time was $277 \pm 118 \mathrm{~min}$, and the effective rate of opening blood vessels was $90.9 \%$. For blood vessels less than $2.0 \mathrm{~mm}$ in diameter, Solitaire AB stents could not be used [35].

Mechanical fragmentation uses the mechanical cutting action of micro-guide wires and micro-catheters to directly fragment some non-mechanized thrombus, which can accelerate the recanalization of occluded blood vessels. Micro-guide wires and catheter thrombi can be applied to small blood vessels less than $1 \mathrm{~mm}$, but mechanical thromboembolism may leave some thrombotic plaques, and cerebral infarction may still occur if the blood vessels are not completely reconnected.

Angioplasty can be divided into balloon-expandable and self-expanding. The use of vascular stenting in patients with obvious vascular stenosis can significantly improve the recanalization rate.

Bridging treatment refers to a series of combined treatments such as arterial thrombolysis and stent thrombectomy in the case of poor effect after IVT. For embolization vessels that cannot be opened due to repeated advance and retreat of guide wire and catheter during mechanical thrombolysis, balloon dilatation technique can be directly applied, and stent implantation can be considered for the treatment of severe residual stenosis. However, whether mechanical thromboembolism, balloon dilatation angioplasty, or stent angioplasty, the broken thrombus may be impacted to the distal end. Postoperative infusion of thrombolytic drugs, the broken thrombus can be quickly decomposed to avoid thrombosis and reduce the incidence of secondary cerebral embolism [36, 37].

Early thrombolysis and endovascular treatment can reopen the blood vessels and restore local blood perfusion, which can save the ischemic endangered brain tissue and reduce mortality and disability. Thrombolytic therapy has become the preferred treatment for acute cerebral infarction.

However, recanalization of the artery may not cause reperfusion of brain tissue. On the other hand, sudden brain tissue reperfusion can also be harmful, leading to blood-brain barrier disruption, hemorrhagic transformation and large-area cerebral edema, which is called "reperfusion injury". Studies have shown that the earlier IVT or intravascular treatment begins and completes within the time window, the higher the success rate and fewer complications. The failure rate increases by $3 \%$ for each $1 \mathrm{~h}$ delay from onset to onset of puncture. For every $1 \mathrm{~h}$ delay from onset to perfusion, the failure rate increases by $7 \%[38,39]$.

\section{Current Challenges}

How to choose the appropriate treatment plan to rescue the IP in the shortest time, in order to effectively target the individualized treatment of patients, which requires the clinician to compare the treatment time window, efficacy, safety and prognosis of different thrombolysis methods, and familiar with and master the relevant treatment processes and techniques. In order to find individualized thrombolytic treatment methods, so that more patients can benefit from it, this is the current research hotspot [40-43].

However, the proportion of thrombolytic therapy in patients with AIS is low. Kaste [44] reported that only 1.6-2.7\% of AIS patients in US community hospitals received thrombolytic therapy, and even in stroke treatment centers of teaching hospitals or large hospitals, the thrombolytic rate was only 4.1$6.3 \%$. According to the New Zealand National Stroke Network data, in 8,857 patients with ischemic and undefined stroke, altplase was administered to 623 patients, and the thrombolytic rate was 7.0\% [45]. Scherf et al analyzed the data of 121,887 stroke admissions in 65 hospitals in the Netherlands, and found that the national average thrombolysis rate increased from $6.402 \%$ to $14.602 \%$ [46]. Qiang et al reported that the use of tissue plasminogen activator in China was only about $1.6 \%$ in the real world. In conclusion, it is urgent to improve thrombolysis rate [47].

\section{Main Reasons for Low Thrombolysis Rate}

First, on the patient side, 1) patients lack medical knowledge and do not know how to recognize the symptoms of acute stroke; 2) some patients do not go to the hospital as soon as possible after onset, resulting in a delay in the hospital; 3) patients are unable to determine the specific onset time; 4) patients are worried about risks, unable to clearly understand the benefits and safety of thrombolysis, unwilling to sign; 5) dif- 
ferences in age, gender, culture and race, and family financial status. Second, on the hospital side, 1) the first doctor lacks professional knowledge of acute stroke; 2) green channel is not smooth enough; 3) some emergency centers lack SUs; 4) long waiting time for blood glucose measurement, blood routine, blood coagulation function and electrocardiogram [48, 49]. Third, on the doctor's side, 1) concerned about complications such as hemorrhagic transformation (HT) after thrombolysis; 2) some doctors are not familiar with the thrombolytic process.

Currently, thrombolytic model is widely used in the treatment of patients with cerebral infarction under the guidance of time window. Multiple factors can affect the time window of thrombolytic treatment, such as the type of cerebral infarction, collateral circulation state, clinical condition, body temperature and cerebral metabolic rate, and so on. At present, it is found that patients with infarction of basilar artery, posterior circulation of brain and anterior circulation with better collateral circulation can extend the time window of thrombolysis appropriately. There are also large differences in the time window of thrombolytic therapy in different individuals. In some patients, the IP has disappeared completely only after $2 \mathrm{~h}$ of onset, while in other patients, most of the IP still exists even after $9 \mathrm{~h}$ of onset. Therefore, it is urgent to explore a new thrombolytic therapy window mode. Previous studies have used image-based pathophysiological window as the guidance mode to evaluate the efficacy and safety of thrombolysis $[50,51]$.

\section{Establishing Clinical Rescue Decision for AIS}

Early revascularization is an important treatment strategy for AIS. Combined application of multiple treatment methods can improve the therapeutic effect. Then: 1) How to improve the popular science efficiency of AIS treatment; 2) How to coordinate the seamless connection of clinical departments and shorten DNT; 3) How to explore the IP over time window; 4) How to predict the risk of hemorrhagic transformation and reocclusion after thrombolysis. We present the following clinical rescue decisions.

When patients with suspected acute stroke come to the hospital, whether they are from outpatient clinic or emergency department, it is necessary to immediately enter the green channel for stroke treatment, conduct National Institutes of Health stroke scale (NIHSS) score, blood pressure and blood glucose monitoring, urgently check craniocerebral computed tomography (CT), platelets and coagulation function, and quickly establish venous channels.

If the CT shows cerebral hemorrhage, neurosurgical consultation should be urgently requested. For patients with surgical indications, drilling and drainage should be performed immediately to remove hematoma. For those without surgical indications, comprehensive treatment can be performed in the SU.

If no cerebral hemorrhage is seen on CT, the diagnosis of ischemic stroke is considered and the stroke treatment team is immediately notified. If the onset time within $4.5 \mathrm{~h}$ and there are no contraindications, IVT (rt-PA $0.9 \mathrm{mg} / \mathrm{kg}$ ) is given immediately. After thrombolysis, if the NIHSS score decreases, it indicates that thrombolysis is effective and the patient is transferred to the SU for further treatment. If the symptoms do not improve, immediate bridging therapy (arterial thrombolysis, stent thrombolysis and other intravascular interventional therapy) is required. If the onset time is greater than $4.5 \mathrm{~h}$ or the exact onset time is unknown, then computed tomography perfusion (CTP), DWI and SWI screening for patients with IP can be immediately treated with arterial thrombolysis, stent thrombectomy, etc.; if there is no IP, then the patient can be transferred directly to the SU.

After treatment, if the patient has no discomfort, the craniocerebral CT examination can be conducted $24 \mathrm{~h}$ later to find out whether there is bleeding. NIHSS score should be performed for $2 \mathrm{~h}, 6 \mathrm{~h}, 24 \mathrm{~h}$ and 7 days after thrombolysis to observe the changes of the disease, and mRs will be used to evaluate the limb recovery of the patient.

After treatment, if the patient has headache, vomiting, gingiva bleeding and other conditions, it is necessary to be alert to the possibility of bleeding transformation, and immediately review the brain $\mathrm{CT}$. If there is no bleeding transformation, it is necessary to continue to observe the changes of the disease. If there is bleeding transformation, neurosurgery department and blood transfusion department should be consulted immediately, and cross matching should be prepared if necessary. Surgical treatment should be carried out immediately if there is an operative pointer. If there is no operation pointer, the dynamic changes of blood pressure and coagulation function should be closely detected, and cryoprecipitation, platelets, plasma, vitamin K1, tranexamic acid, etc. should be input if necessary. Whether surgical treatment is performed or not, it is necessary to transfer to the neurological intensive care unit (ICU) for comprehensive treatment (Fig. 1).

In order to improve the safety and effectiveness of thrombolysis, we propose strategies including: preparation before thrombolysis, observation during thrombolysis and response after thrombolysis. The specific operations are as follows.

\section{Before thrombolysis}

First of all, in terms of popular science, the awareness rate of the people should be increased, by using the media platforms such as TV, radio, WeChat and brochures to expand the scope and depth of stroke science, and make FAST deeply popular. Improve the people's understanding of the necessity of thrombolysis, time urgency and safety, and fully understand that the benefits of early thrombolysis far outweigh the risks $[52,53]$. Secondly, at the hospital level, 1) popularize the knowledge of cerebral infarction, improve the awareness of early thrombolytic therapy for patients and medical staff; 2) establish a referral mechanism for network hospitals; 3) open a green channel for thrombolytic therapy in patients with acute ischemic cerebral infarction; 4) train relevant professionals to improve clinical execution; 5) take a door-to-needle time (DNT) thrombolysis management system to shorten or eliminate in-hospital delay time and improve the response rate of thrombolytic therapy $[54,55]$. Third, in terms of doctors, 1) fully understand the importance of thrombolysis in AIS; 2) quickly identify patients with acute stroke, and grasp the emergency thrombolysis 


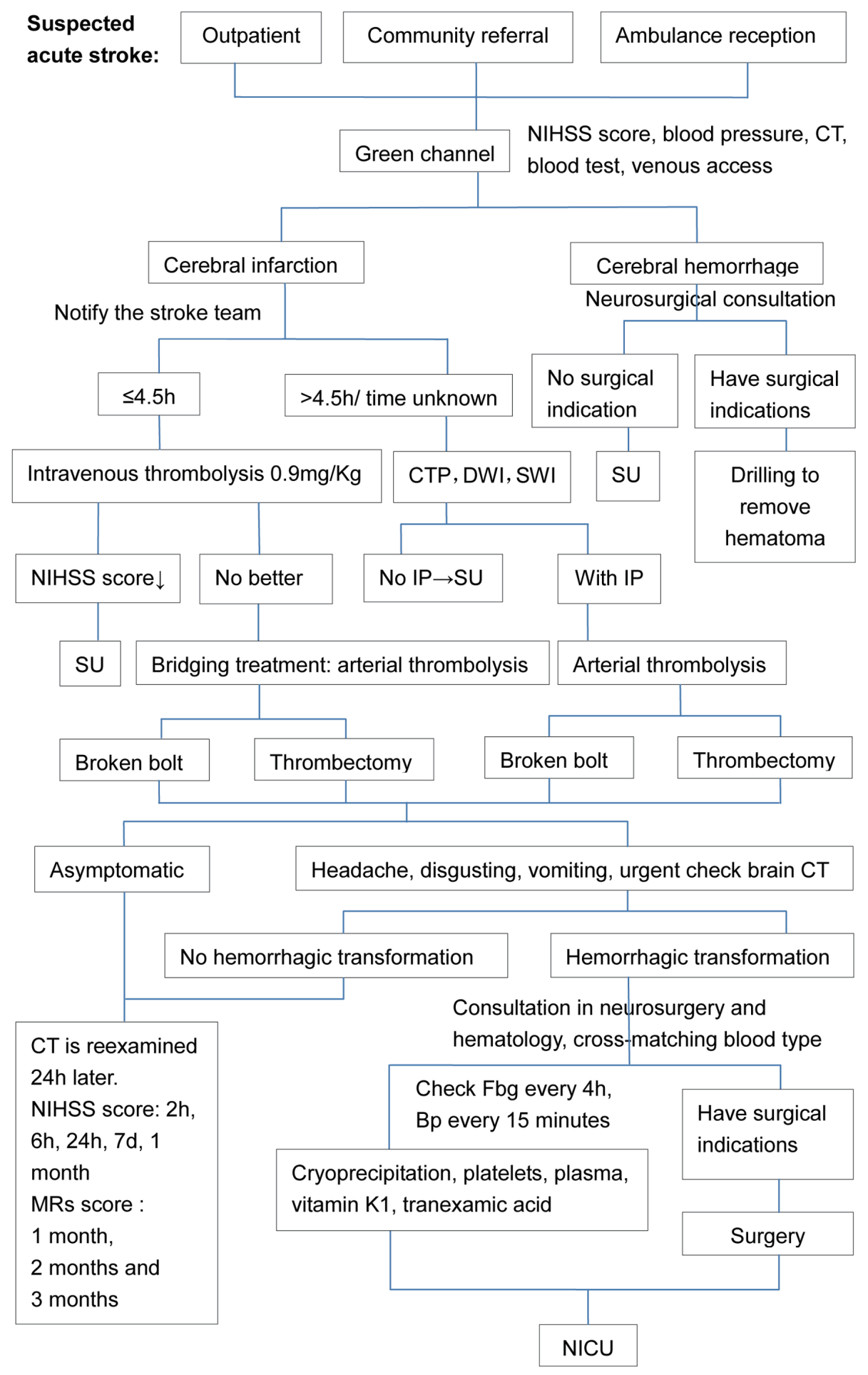

Figure 1. Acute stroke treatment strategy process.

time window, indications and contraindications; 3) for patients with unknown onset time, IP can be determined by imaging; 4) be skilled in arterial thrombolysis and endovascular treatment and other related technologies, and can successfully complete bridging treatment; 5) can have the ability to cope with hemor- rhagic transformation after thrombolysis.

The proportion of thrombolytic therapy used by clinicians is relatively low, most of which is due to the fear that hemorrhagic transformation will aggravate the symptoms of neurological deficits and increase the mortality rate. In order to en- 
sure the safety and effectiveness of thrombolysis, according to the January 2018 AHA/ASA issued "2018 Early Management Guide for Acute Ischemic Stroke" [56], it is important to clearly identify patients with indications and contraindications.

Early identification of patients at high risk of bleeding is an important issue for clinicians. In order to effectively predict the risk of hemorrhagic transformation, in addition to routine examination of blood routine, blood glucose, liver and kidney function, myocardial zymogram, coagulation function, electrocardiogram, etc., if necessary, also interleukin-6 (IL-6), tumor necrosis factor- $\alpha$ (TNF- $\alpha$ ) and matrix metalloproteinase- 9 (MMP-9) should be detected, homocysteine (HCY), C-reactive protein (CRP) and other factors associated with hemorrhagic transformation and blood-brain barrier disruption and microvascular injury should be checked to assess the risk of hemorrhagic transformation. More importantly, patients with intracranial hypertension, hyperglycemia, high blood pressure, electrolyte and acid-base balance disorders, dysphagia and urinary system diseases require timely symptomatic supportive care. For patients with AIS with unknown onset time, head CT excludes cerebral hemorrhage, and the mismatch between DWI and PWI is more than $20 \%$, which can be considered as the pathophysiological basis of IP. Even beyond the traditional thrombolytic treatment time window, thrombolytic therapy for these patients can still improve the blood perfusion in the penumbra, which is conducive to the further recovery of nerve function.

\section{In the process of thrombolysis}

Patients with AIS within $3 \mathrm{~h}$ (level I recommendation, level A evidence) and 3 - $4.5 \mathrm{~h}$ (level I recommendation, level B evidence) will be given rt-PA thrombolysis as soon as possible. The use of rt-PA is $0.9 \mathrm{mg} / \mathrm{kg}$ (maximum dose is $90 \mathrm{mg}$ ) intravenous drip, maintained for $1 \mathrm{~h}, 10 \%$ of which is intravenously injected within $1 \mathrm{~min}$, and patients should be closely monitored during medication and within $24 \mathrm{~h}$ (level I recommendation, level A evidence) [56]. Blood pressure measurements and neurologic function assessments are performed every $15 \mathrm{~min}$ during and after IVT therapy, and then once every 30 min for 6 $\mathrm{h}$, once every hour thereafter until $24 \mathrm{~h}$ after treatment.

If systolic blood pressure $\geq 180 \mathrm{~mm} \mathrm{Hg}$ or diastolic blood pressure $\geq 100 \mathrm{~mm} \mathrm{Hg}$, the frequency of blood pressure monitoring should be increased, and antihypertensive drugs should be given. Nasal feeding tubes, catheters and intra-arterial pressure measuring tubes should be delayed in case of permission [57].

If IVT is not successful, immediate use of bridging therapy can increase the success rate of treatment [58]. Arterial thrombolysis is performed with $10 \mathrm{~mL}$ local anesthesia with $1 \%$ lidocaine, and the responsible vessel is identified by digital subtraction angiography (DSA) examination. Seldinger technology punctures the unilateral femoral artery, inserts the $6 \mathrm{~F}$ catheter sheath, and then inserts the $6 \mathrm{~F}$ catheter (or balloon catheter) into the responsible artery under guided wire guidance. The microcatheter is inserted into the proximal end of occluded vessels or to the distal end of the thrombus under the guidance of the microcatheter, injecting with urokinase
10,000 U/min (maximum 1 million units) or rt-PA $1 \mathrm{mg} / \mathrm{min}$; withdraw catheter buried in the thrombus, inject $100,000 \mathrm{U}$ of urokinase; withdraw the catheter to the proximal end of the thrombus, and inject 100,000 U of urokinase. During the operation, the angiography is performed once every $10 \mathrm{~min}$. If the angiographic stenosis rate after lysis is $\geq 70 \%$, stent placement is performed.

If arterial thrombolysis is still ineffective, mechanical thrombolysis can be taken. First, the micro-guide wire is used to slowly advance from the occluded blood vessel stump to the distal end. After the micro-guide wire is completely passed, the micro-catheter is followed, and the advancing and retreating is repeated two or three times to break up the thrombus. DSA examination confirmed the vascular dredging effect; if the distal blood vessels have been unobstructed, the microcatheter should be used to slowly push rt-PA $10 \mathrm{mg}$. After about 10 min, the micro-guide wire and micro-catheter are reapplied to open the blood vessel.

For patients with obvious thrombus or plaque in the blood vessels, Solitaire AB stent is used to remove the thrombus, release the Solitaire AB stent at the distal end of the thrombus, and pull back to the body. Repeated operations are performed several times to remove the thrombus in whole or in pieces. In the above operation, the rt-PA is continuously pushed, and the total amount is $\leq 30 \mathrm{mg}$. DSA examinations are performed during the procedure until it is determined that the occluded vessels are fully open.

Patients should be closely monitored during IVT and bridging therapy. In case of severe headache, hypertension, nausea or vomiting, or deterioration of neurological function, thrombolytic drugs should be stopped immediately and brain CT examination should be conducted [59].

\section{Treatment after thrombolysis}

After thrombolysis, patients are admitted to the ICU or SU for monitoring. Close observation includes oral mucosa, puncture site, urine color, traumatic site and vomiting. The NIHSS scores before thrombolysis and $24 \mathrm{~h}$ and 7 days after thrombolysis are recorded. CT examination will be conducted at any time for patients with disease changes. If NIHSS score more than four points at baseline is defined as hemorrhagic transformation after thrombolysis, according to the amount of bleeding, whether surgery is needed to do is immediately evaluated; if there is no indication for surgery, cold precipitation, platelets, plasma, vitamin K1, tranexamic acid and other drugs can be used for symptomatic treatment, and the patient's vital signs and NIHSS score changes should be closely monitored [60-62]. If there is no bleeding, anti-platelet aggregation drugs (aspirin $100 \mathrm{mg}$ /day, clopidogrel $75 \mathrm{mg}$ /day) are given $24 \mathrm{~h}$ after surgery. For those with stent implantation, low-molecularweight heparin calcium anticoagulation therapy (subcutaneous injection $0.4 \mathrm{~mL} / \mathrm{day}$, continuous use of 3 days) is required to prevent the formation of thrombus. In order to prevent vasospasm, calcium channel antagonists are given (nimoton $6 \mathrm{~mL} / \mathrm{h}$, adjusted according to blood pressure, 1 - 3 days), to control systolic blood pressure 110 - $140 \mathrm{~mm} \mathrm{Hg}$. In the SU, cause should be found actively, comprehensive treatment should be 
given, and if nerve function deficiency, rehabilitation exercise should be done as soon as possible.

\section{Conclusion}

There are many methods for thrombolytic therapy and endovascular treatment in ischemic stroke. Most patients receiving thrombolytic therapy quickly restore neurological function at an early stage, but the rate of thrombolysis in patients with cerebral apoplexy is still very low, and the reason is no AIS treatment decision-making system research. This article breaks the traditional time-based treatment plan, uses biochemical and imaging indicators to examine the hemorrhagic transformation and blood-brain barrier destruction and microvascular damage closely related factors, and evaluates the penumbra and the risk of hemorrhagic transformation after thrombolysis, establishing a clinical decision for acute stroke with "the thrombolysis mode under the guidance of time window" combined with the "thrombotic mode under the guidance of pathophysiology window", to explore the opportunity of reperfusion treatment for patients who have exceeded the time window but still have the IP, which is conducive to the recovery of neurological function. The treatment of hemorrhagic transformation after thrombolysis is proposed with clear countermeasures, and the treatment plan and clinical strategy for acute stroke are systematically expounded, which can improve the safety and effectiveness of thrombolysis.

\section{Acknowledgments}

We are grateful to colleagues in the field of acute ischemic stroke.

\section{Financial Disclosure}

This article is a clinical review, no funding.

\section{Conflict of Interest}

On behalf of all authors, the corresponding author states that there is no conflict of interest.

\section{Author Contributions}

YB and XYD: literature collection; YB, MZ, RA and JX: literature analysis; YB, XYD and MZ: manuscript preparation; YB, XDL and GJL: reviewing of manuscript.

\section{References}

1. Pan A, Sun Q, Okereke OI, Rexrode KM, Hu FB. Depression and risk of stroke morbidity and mortal- ity: a meta-analysis and systematic review. JAMA. 2011;306(11):1241-1249.

2. Favate AS, Younger DS. Epidemiology of ischemic stroke. Neurol Clin. 2016;34(4):967-980.

3. Truelsen T, Krarup LH, Iversen HK, Mensah GA, Feigin VL, Sposato LA, Naghavi M. Causes of Death Data in the Global Burden of Disease Estimates for Ischemic and Hemorrhagic Stroke. Neuroepidemiology. 2015;45(3):152-160.

4. Paciaroni M, Balucani C, Agnelli G, Caso V, Silvestrelli G, Grotta JC, Demchuk AM, et al. Systemic thrombolysis in patients with acute ischemic stroke and Internal Carotid ARtery Occlusion: the ICARO study. Stroke. 2012;43(1):125-130.

5. Fukuoka T, Nakazato Y, Kawasaki H, Ikeda K, Furuya T, Miyake A, Mitsufuji T, et al. The clinical features of ischemic stroke patients for whom smoking was considered the sole risk factor for ischemic stroke. Intern Med. 2018;57(12):1703-1706.

6. Colivicchi F, Bassi A, Santini M, Caltagirone C. Discontinuation of statin therapy and clinical outcome after ischemic stroke. Stroke. 2007;38(10):2652-2657.

7. Ji R, Schwamm LH, Pervez MA, Singhal AB. Ischemic stroke and transient ischemic attack in young adults: risk factors, diagnostic yield, neuroimaging, and thrombolysis. JAMA Neurol. 2013;70(1):51-57.

8. Putaala J, Metso TM, Metso AJ, Makela E, Haapaniemi E, Salonen O, Kaste M, et al. Thrombolysis in young adults with ischemic stroke. Stroke. 2009;40(6):20852091.

9. Vannucci RC, Vannucci SJ. Glucose, acidosis, and perinatal hypoxic-ischemic brain damage. Developmental Disabilities Research Reviews. 2015;3:69-75.

10. Seifert G, Schilling K, Steinhauser C. Astrocyte dysfunction in neurological disorders: a molecular perspective. Nat Rev Neurosci. 2006;7(3):194-206.

11. Saver JL. Time is brain - quantified. Stroke. 2006;37(1):263-266.

12. Audebert HJ, Sobesky J. Stroke: 'time is brain' after stroke, regardless of age and severity. Nat Rev Neurol. 2014;10(12):675-676.

13. Donnan GA, Davis SM. Neuroimaging, the ischaemic penumbra, and selection of patients for acute stroke therapy. Lancet Neurol. 2002;1(7):417-425.

14. Zerna C, Thomalla G, Campbell BCV, Rha JH, Hill MD. Current practice and future directions in the diagnosis and acute treatment of ischaemic stroke. Lancet. 2018;392(10154):1247-1256.

15. Campbell BC, Mitchell PJ, Kleinig TJ, Dewey HM, Churilov L, Yassi N, Yan B, et al. Endovascular therapy for ischemic stroke with perfusion-imaging selection. N Engl J Med. 2015;372(11):1009-1018.

16. Meoded A, Poretti A, Benson JE, Tekes A, Huisman TA. Evaluation of the ischemic penumbra focusing on the venous drainage: the role of susceptibility weighted imaging (SWI) in pediatric ischemic cerebral stroke. J Neuroradiol. 2014;41(2):108-116.

17. Catanese L, Tarsia J, Fisher M. Acute Ischemic Stroke Therapy Overview. Circ Res. 2017;120(3):541-558. 
18. Agarwal S, Warburton EA, Baron JC. From Time is brain to Physiology is brain: a case for reflection in acute stroke treatment decisions. Brain. 2015;138(Pt 7):1768-1770.

19. Fisher M, Saver JL. Future directions of acute ischaemic stroke therapy. Lancet Neurol. 2015;14(7):758-767.

20. Feigin VL, Norrving B, George MG, Foltz JL, Roth GA, Mensah GA. Prevention of stroke: a strategic global imperative. Nat Rev Neurol. 2016;12(9):501-512.

21. Mueller L, Pult F, Meisterernst J, Heldner MR, Mono ML, Kurmann R, Buehlmann M, et al. Impact of intravenous thrombolysis on recanalization rates in patients with stroke treated with bridging therapy. Eur J Neurol. 2017;24(8):1016-1021.

22. Deb P, Sharma S, Hassan KM. Pathophysiologic mechanisms of acute ischemic stroke: An overview with emphasis on therapeutic significance beyond thrombolysis. Pathophysiology. 2010;17(3):197-218.

23. Awadh M, MacDougall N, Santosh C, Teasdale E, Baird T, Muir KW. Early recurrent ischemic stroke complicating intravenous thrombolysis for stroke: incidence and association with atrial fibrillation. Stroke. 2010;41(9):19901995.

24. Kim JS, Kim YJ, Lee KB, Cha JK, Park JM, Hwang Y, Kim EG, et al. Low- versus standard-dose intravenous alteplase in the context of bridging therapy for acute ischemic stroke: a Korean enchanted study. J Stroke. 2018;20(1):131-139.

25. Gerschenfeld G, Muresan IP, Blanc R, Obadia M, Abrivard M, Piotin M, Alamowitch S. Two Paradigms for Endovascular Thrombectomy After Intravenous Thrombolysis for Acute Ischemic Stroke. JAMA Neurol. 2017;74(5):549-556.

26. Ahmed N, Wahlgren N, Brainin M, Castillo J, Ford GA, Kaste M, Lees KR, et al. Relationship of blood pressure, antihypertensive therapy, and outcome in ischemic stroke treated with intravenous thrombolysis: retrospective analysis from Safe Implementation of Thrombolysis in Stroke-International Stroke Thrombolysis Register (SITS-ISTR). Stroke. 2009;40(7):2442-2449.

27. Nakagawara J, Minematsu K, Okada Y, Tanahashi N, Nagahiro S, Mori E, Shinohara Y, et al. Thrombolysis with $0.6 \mathrm{mg} / \mathrm{kg}$ intravenous alteplase for acute ischemic stroke in routine clinical practice: the Japan post-Marketing Alteplase Registration Study (J-MARS). Stroke. 2010;41(9):1984-1989

28. Ma QF, Chu CB, Song HQ. Intravenous versus intra-arterial thrombolysis in ischemic stroke: a systematic review and meta-analysis. PLoS One. 2015;10(1):e0116120.

29. Moonis M. Intraarterial thrombolysis within the first three hours after acute ischemic stroke in selected patients. Stroke. 2009;40(7):2611-2612.

30. Mattle HP, Arnold M, Georgiadis D, Baumann C, Nedeltchev K, Benninger D, Remonda L, et al. Comparison of intraarterial and intravenous thrombolysis for ischemic stroke with hyperdense middle cerebral artery sign. Stroke. 2008;39(2):379-383.

31. Gocmen R, Arsava EM, Oguz KK, Topcuoglu MA. Atherosclerotic intracranial internal carotid artery calcification and intravenous thrombolytic therapy for acute is- chemic stroke. Atherosclerosis. 2018;270:89-94.

32. Daffertshofer M, Gass A, Ringleb P, Sitzer M, Sliwka U, Els T, Sedlaczek O, et al. Transcranial low-frequency ultrasound-mediated thrombolysis in brain ischemia: increased risk of hemorrhage with combined ultrasound and tissue plasminogen activator: results of a phase II clinical trial. Stroke. 2005;36(7):1441-1446.

33. Auboire L, Sennoga CA, Hyvelin JM, Ossant F, Escoffre JM, Tranquart F, Bouakaz A. Microbubbles combined with ultrasound therapy in ischemic stroke: A systematic review of in-vivo preclinical studies. PLoS One. 2018;13(2):e0191788.

34. Shireman TI, Wang K, Saver JL, Goyal M, Bonafe A, Diener HC, Levy EI, et al. Cost-effectiveness of solitaire stent retriever thrombectomy for acute ischemic stroke: results from the SWIFT-PRIME trial (Solitaire with the intention for thrombectomy as primary endovascular treatment for acute ischemic stroke). Stroke. 2017;48(2):379-387.

35. Roth C, Junk D, Papanagiotou P, Keuler A, Korner H, Schumacher M, Reith W. A comparison of 2 stroke devices: the new Aperio clot-removal device and the solitaire AB/FR. AJNR Am J Neuroradiol. 2012;33(7):13171320.

36. Mazighi M, Meseguer E, Labreuche J, Amarenco P. Bridging therapy in acute ischemic stroke: a systematic review and meta-analysis. Stroke. 2012;43(5):1302-1308.

37. Saver JL, Goyal M, van der Lugt A, Menon BK, Majoie CB, Dippel DW, Campbell BC, et al. Time to treatment with endovascular thrombectomy and outcomes from ischemic stroke: a meta-analysis. JAMA. 2016;316(12):1279-1288.

38. Cho AH, Cho YP, Lee DH, Kwon TW, Kwon SU, Suh $\mathrm{DC}$, Kim JS, et al. Reperfusion injury on magnetic resonance imaging after carotid revascularization. Stroke. 2014;45(2):602-604.

39. Liu P, Zhao H, Wang R, Wang P, Tao Z, Gao L, Yan F, et al. MicroRNA-424 protects against focal cerebral ischemia and reperfusion injury in mice by suppressing oxidative stress. Stroke. 2015;46(2):513-519.

40. Scheitz JF, Seiffge DJ, Tutuncu S, Gensicke H, Audebert HJ, Bonati LH, Fiebach JB, et al. Dose-related effects of statins on symptomatic intracerebral hemorrhage and outcome after thrombolysis for ischemic stroke. Stroke. 2014;45(2):509-514.

41. Tsivgoulis G, Zand R, Katsanos AH, Turc G, Nolte $\mathrm{CH}$, Jung S, Cordonnier C, et al. Risk of symptomatic intracerebral hemorrhage after intravenous thrombolysis in patients with acute ischemic stroke and high cerebral microbleed burden: a meta-analysis. JAMA Neurol. 2016;73(6):675-683.

42. Bongiorno DM, Daumit GL, Gottesman RF, Faigle R. Comorbid psychiatric disease is associated with lower rates of thrombolysis in ischemic stroke. Stroke. 2018;49(3):738-740.

43. Reiff T, Michel P. Reasons and evolution of nonthrombolysis in acute ischaemic stroke. Emerg Med J. 2017;34(4):219-226.

44. Kaste M. Thrombolysis: what more does it take? Stroke. 2005;36(2):200-202. 
45. Liu Q, Ranta AA, Abernethy G, Barber PA. Trends in New Zealand stroke thrombolysis treatment rates. N Z Med J. 2017;130(1453):50-56.

46. Scherf S, Limburg M, Wimmers R. Increase in national intravenous thrombolysis rates for ischaemic stroke between 2005 and 2012: is bigger better? Bmc Neurology. 2016;16:1-6.

47. Dong Q, Dong Y, Liu L, Xu A, Zhang Y, Zheng H, Wang Y. The Chinese Stroke Association scientific statement: intravenous thrombolysis in acute ischaemic stroke. Stroke Vasc Neurol. 2017;2(3):147-159.

48. Fonarow GC, Zhao X, Smith EE, Saver JL, Reeves MJ, Bhatt DL, Xian Y, et al. Door-to-needle times for tissue plasminogen activator administration and clinical outcomes in acute ischemic stroke before and after a quality improvement initiative. JAMA. 2014;311(16):1632-1640.

49. Kamal N, Holodinsky JK, Stephenson C, Kashayp D, Demchuk AM, Hill MD, Vilneff RL, et al. Improving door-to-needle times for acute ischemic stroke: effect of rapid patient registration, moving directly to computed tomography, and giving alteplase at the computed tomography scanner. Circ Cardiovasc Qual Outcomes. 2017;10(1):e003242.

50. de Havenon A, Southerland AM. In large vessel occlusive stroke, time is brain... but collaterals are time. Neurology. 2018;90(4):153-154.

51. Bivard A, Parsons M. Tissue is more important than time: insights into acute ischemic stroke from modern brain imaging. Current Opinion in Neurology. 2017;31:1.

52. Aroor S, Singh R, Goldstein LB. BE-FAST (Balance, Eyes, Face, Arm, Speech, Time): reducing the proportion of strokes missed using the FAST mnemonic. Stroke. 2017;48(2):479-481.

53. Wilson D, Charidimou A, Ambler G, Fox ZV, Gregoire $\mathrm{S}$, Rayson P, Imaizumi T, et al. Recurrent stroke risk and cerebral microbleed burden in ischemic stroke and TIA: A meta-analysis. Neurology. 2016;87(14):1501-1510.

54. Kamal N, Sheng S, Xian Y, Matsouaka R, Hill MD, Bhatt
DL, Saver JL, et al. Delays in door-to-needle times and their impact on treatment time and outcomes in get with the guidelines-stroke. Stroke. 2017;48(4):946-954.

55. Jacoby JS, Draper HM, Dumkow LE, Farooq MU, DeYoung GR, Brandt KL. Emergency medicine pharmacist impact on door-to-needle time in patients with acute ischemic stroke. Neurohospitalist. 2018;8(2):60-65.

56. Furie KL, Jayaraman MV. 2018 guidelines for the early management of patients with acute ischemic stroke. Stroke. 2018;49(3):509-510.

57. Hemmen TM, Raman R, Guluma KZ, Meyer BC, Gomes JA, Cruz-Flores S, Wijman CA, et al. Intravenous thrombolysis plus hypothermia for acute treatment of ischemic stroke (ICTuS-L): final results. Stroke. 2010;41(10):22652270 .

58. Bellwald S, Weber R, Dobrocky T, Nordmeyer H, Jung S, Hadisurya J, Mordasini P, et al. Direct mechanical intervention versus bridging therapy in stroke patients eligible for intravenous thrombolysis: a pooled analysis of 2 registries. Stroke. 2017;48(12):3282-3288.

59. Fransen PS, Berkhemer OA, Lingsma HF, Beumer D, van den Berg LA, Yoo AJ, Schonewille WJ, et al. Time to reperfusion and treatment effect for acute ischemic stroke: a randomized clinical trial. JAMA Neurol. 2016;73(2):190196.

60. Raposo N, Curtze S. Cerebral microbleeds in acute ischemic stroke: A red flag for IV thrombolysis. Neurology. 2016;87(15):1526-1527.

61. Yaghi S, Willey JZ, Cucchiara B, Goldstein JN, Gonzales NR, Khatri P, Kim LJ, et al. Treatment and outcome of hemorrhagic transformation after intravenous alteplase in acute ischemic stroke: a scientific statement for healthcare professionals from the American Heart Association/ American Stroke Association. Stroke. 2017;48(12):e343e361.

62. Bao Y, Ding Y, Liu Q, et al. Clinical features of a largearea cerebral infarction with good prognosis. Journal of Medical Cases. 2019;10:141-145. 